|| ISSN(online): 2589-8698 || ISSN(print): 2589-868X || International Journal of Medical and Biomedical Studies

Available Online at www.ijmbs.info

PubMed (National Library of Medicine ID: 101738825)

Index Copernicus Value 2017: 40.03

\title{
EVALUATION OF GALECTIN-3 LEVELS IN OBESE ADOLESCENTS
}

\author{
Huseyin Dag', Nevin Cetin Dag1, Emine Turkkan', Ebru Misirli Ozdemir', Okan Dikker² \\ ${ }^{1}$ University of Health Sciences, Istanbul Okmeydani Training and Research Hospital, Department of \\ Pediatrics, Istanbul, Turkey \\ ${ }^{2}$ University of Health Sciences, Istanbul Okmeydani Training and Research Hospital, Department of \\ Medical Biochemistry, Istanbul, Turkey
}

Article Info: Received 15 February 2019; Accepted 02 March. 2019

Cite this article as: Dag, H., Dag, N., Turkkan, E., Ozdemir, E., \& Dikker, O. (2019). EVALUATION OF GALECTIN-3 LEVELS IN OBESE ADOLESCENTS. International Journal of Medical and Biomedical Studies, 3(3).

DOI: https://doi.org/10.32553/ijmbs.v3i3.130

Address for Correspondence: Dr. Hüseyin DAG, Ministry of Health, Istanbul Okmeydani Training and Research Hospital, Department of Pediatrics, Istanbul, Turkey

Conflict of interest: No conflict of interest.

\section{Abstract}

Background: Galectin-3 is a member of the family of soluble beta-galactoside binding lectins, which is involved in inflammation, cell differentiation, adhesion, proliferation, angiogenesis and cancer cell invasion. Increased galectin-3 levels in obese patients have been shown in previous studies. In our study; we aimed to investigate the galectin-3 levels in obese adolescents for the first time in the literature.

Materials and methods: A total of 85 adolescent patients who were admitted to our pediatrics outpatient clinic between December $19^{\text {th }}$ of 2018 and January $31^{\text {st }}$ of 2019 were included in our study. Among those patients 52 were obese and 33 were normal-weighted. Adolescents having body mass index (BMI) 95\% percentile and above according to their age and sex were defined as obese. EnzymeLinked Immunosorbent Assay kit was used for the measurement of galectin-3 in serum. Laboratory tests (Galectin-3, HbA1c, glucose, insulin, HOMA-IR), sex, age and BMI were statistically compared between groups.

Results: We found no statistically significant difference in terms of gender distribution rates, age, glucose or galectin-3 levels among obese adolescent and healthy control groups ( $\mathrm{p}>0.05)$. The BMI, HbA1c, HOMA-IR and insulin levels of the obese adolescent group were found to be significantly higher than the healthy control group $(p<0.05)$. We found no statistically significant correlation between galectin-3 levels and other parameters in obese adolescent group ( $p>0.05)$.

Conclusion: In our study, we did not find any relationship between serum galectin-3 levels and obesity in adolescents. We believe that the effects of galectin-3 levels on obesity in adolescents should be more clearly explained by further investigations, which also measures other biomarkers of the mechanism of action.

Keywords: Galectin-3, obesity, adolescent

List of abbreviations: BMI: Body-Mass Index, CV: Variation coefficient, ELISA: Enzyme linked immunoassay, HOMA-IR: Homeostatic model of assessment-insulin resistance, IL: Interleukin, $r$ : Correlation coefficient, SD: Standard deviation. 


\section{Introduction:}

Galectin-3 is a member of the family of soluble beta-galactoside binding lectins, which is involved in inflammation, cell differentiation, adhesion, proliferation, angiogenesis and cancer cell invasion (1). The addition of recombinant galectin-3 in vitro induces preadipocyte proliferation (2). Extracellular galectin-3 stimulates apoptosis, while galectin-3 in intracellular compartments protects against apoptosis (3). Galectin-3, produced in various cell types such as epithelial cells, macrophages and adipocytes, is present in every part of the cell. Extracellular galectin-3 plays a role in the relationships between epithelial cells and extracellular matrix (4).

Increased levels of galectin-3 in obese patients have been shown in previous studies (5-7). Various mechanisms have been described before in this increase such as the defected adiponectin activity in obesity eliminates the inhibitory effect and causes an increase in galectin-3 levels or elevated interleukin-6 (IL-6) levels may also increase the galectin-3 levels $(5,6)$. Some studies have reported that galectin-3 plays a protective role in obesity-related metabolic complications and inflammation $(8,9)$.

There is a low-grade inflammation due to the increase of proinflammatory cytokine levels in obesity characterized by excessive accumulation of lipids and adipose tissue that causes ectopic fat accumulation in different tissues $(10,11)$. In this study; we aimed to evaluate the levels of galectin-3 in obese adolescents and to investigate their correlations with other parameters.

\section{Material and method:}

A total of 85 adolescent patients aged between 10 and 16 years, who were admitted to our pediatrics outpatient clinic between December $19^{\text {th }}$ of 2018 and January $31^{\text {st }}$ of 2019 were included in othis study. Among those patients 52 were obese and 33 were normal-weighted. Body mass index (BMI) was obtained by division of weight of the patient in $\mathrm{kg}$ to the square of the height in meters. Adolescents having BMI 95\% percentile and above according to their age and sex were defined as obese (12). For insulin resistance, Homeostatic model of assessment $\mathrm{mg}$ (HOMA-IR) was calculated with the (insulin IU / L $x$ glucose $\mathrm{mg} / \mathrm{dL}$ ) / 405 formula (13). The obese patients with HOMA-IR values of higher than 2.5 were considered to be obese adolescents with insulin resistance and those with a value of 2.5 or less were evaluated as adolescents without insulin resistance.

In obese and control groups, smokers, patients with chronic diseases, infection, metabolic and endocrinologic diseases, those who received drug treatments such as corticosteroids and those with malignancy were not included in the study. In addition, adolescents having normalweight but insulin resistance were not included in the healthy control group.

The patients who were observed to be in compliance with the inclusion criteria were informed about the study (in addition to routine examinations, an additional 1 tube of blood will be taken for the study). After obtaining informed consent form from the volunteers who wanted to participate in the study, detailed medical histories of the participants were achieved. From the volunteers, while obtaining blood for their routine examinations, only one more biochemistry tube blood was taken for the study. The serum was kept at room temperature for 20 minutes, and then the blood tube was centrifuged at $4000 \mathrm{rpm}$ for 10 minutes and stored at $-80^{\circ} \mathrm{C}$.

On the day of analysis, sera were allowed to dissolve at room temperature. Enzyme-Linked Immunosorbent Assay (ELISA) kits were used for the measurement of Galectin-3 levels in serum (Human Galectin-3 ELISA, Bioassay Technology Laboratory Lot No: E1811005). The analytical (linear) measurement range was 5 - 2000 pg / mL for galectin-3. The minimal detection limit was $2.49 \mathrm{pg} \mathrm{/} \mathrm{mL}$. The reported intraassay and interassay CV's were $<8 \%$ and $<10 \%$, respectively. 
Hemoglobin A1c (HbA1c) was measured in autoanalyzer (Biorad, Variant II turbo, Japan) with the high performance liquid chromatography method, glucose was measured by colorimetric method, insulin was measured in autoanalyzer with Chemiluminescence immunoassay (Beckman Coulter Brand, AU 5800 model, USA).

Laboratory tests (Galectin-3, HbA1c, glucose, insulin, HOMA-IR), sex, age and BMI were compared between the groups. Correlations between galectin-3 and other laboratory parameters were examined in obese adolescent group.

The present study was approved by the Local Ethics Committee (date:December $18^{\text {th }}$ of 2018; no: 1076) and performed in accordance with the guidelines of the Declaration of Helsinki.

Statistical Analyses: IBM SPSS Statistics 22 (IBM SPSS, Turkey) program wasperformed for the statistical analyses. The fit of the parameters to normal distribution was evaluated by Shapiro Wilks test. In comparison of two groups, Student t-test was performed to compare the parameters with normal distribution and Mann-Whitney $U$ test was used to compare the parameters that did not show normal distribution. Chi Square test and Continuity (Yates) correction were performed to compare qualitative data. Pearson correlation analysis was used to examine the relationships between parameters with normal distribution and Spearman's rho correlation analysis was performed to examine the relationships between parameters which are not compatible with normal distribution. Significance was evaluated as $p<0.05$.

\section{Results:}

The study was carried out with 85 volunteer adolescents, 52 obese patients and 33 healthy control cases with normal weight. Forty three of the obese adolescents were having insulin resistance while 9 did not have insulin resistance. The mean age of the adolescents was $12.83 \pm$ 1.96 years. The mean BMI was $28.28 \pm 5.24 \mathrm{~kg} /$ $\mathrm{m}^{2}$. Demographic and laboratory data of the study groups were compared (Table 1).

We found no statistically significant difference in terms of gender distribution rates, age, glucose or galectin-3 levels among obese adolescent and healthy control groups ( $p>0.05$ ).

The BMI, HbA1c, HOMA-IR and insulin levels of the obese adolescent group were found to be significantly higher than the healthy control group ( $p<0.05)$.

Table 1: Demographic and laboratory data of Obese and Control Adolescent Groups

\begin{tabular}{|c|c|c|c|}
\hline & $\begin{array}{l}\text { Obese Adolescent } \\
\text { Group (n:52) }\end{array}$ & $\begin{array}{l}\text { Healthy Control Adolescent } \\
\text { Group (n:33) }\end{array}$ & \multirow[t]{2}{*}{ p } \\
\hline & Mean士SD & Mean \pm SD & \\
\hline Age (year) & $12.79 \pm 2.04$ & $12.88 \pm 1.84$ & ${ }^{1} 0.848$ \\
\hline \multicolumn{4}{|l|}{ Gender $_{n(\%)}$} \\
\hline female & $20(38.4 \%)$ & $16(48.4 \%)$ & ${ }^{2} 0.495$ \\
\hline male & $32(61.6 \%)$ & $17(51.6 \%)$ & \\
\hline BMI $\left(\mathrm{kg} / \mathrm{m}^{2}\right)$ & $31.29 \pm 3.75$ & $23.29 \pm 3.09$ & ${ }^{1}<0.001^{*}$ \\
\hline Galectin-3 (pg/mL) (median) & $412.85 \pm 616.42(162.2)$ & $527.41 \pm 847.91(122)$ & ${ }^{3} 0.853$ \\
\hline HbA1c (\%) & $5.49 \pm 0.36$ & $5.2 \pm 0.33$ & ${ }^{1} 0.002^{*}$ \\
\hline Glucose (mg/dL) & $89.91 \pm 9.47$ & $87.92 \pm 8.88$ & ${ }^{1} 0.391$ \\
\hline HOMA-IR (median) & $4.08 \pm 2.05(3.6)$ & $1.84 \pm 0.77(1.6)$ & ${ }^{3}<0.001^{*}$ \\
\hline Insulin (IU/L) ${ }_{\text {(median) }}$ & $18.29 \pm 8.58(16.8)$ & $8.35 \pm 3.17(7.8)$ & ${ }^{3}<0.001^{*}$ \\
\hline
\end{tabular}


${ }^{1}$ Student $t$ test $\quad{ }^{2}$ Continuity (Yates) correction $\quad{ }^{3}$ Mann Whitney U test $\quad{ }^{*} p<0.05$

BMI: Body-Mass Index, HOMA-IR: Homeostatic model of assessment-insulin resistance, SD: Standard deviation.

Correlations between galectin-3 and other parameters were evaluated in the obese adolescent group (Table 2). We found no significant correlation between galectin-3 levels and other parameters in obese adolescent group ( $p>0.05)$.

Table 2: Correlations between galectin-3 levels and other parameters in obese adolescent group

\begin{tabular}{|l|l|l|}
\hline & \multicolumn{2}{|l|}{ Galectin-3 levels } \\
\hline & $\mathbf{r}$ & $\mathbf{p}$ \\
\hline BMI & $-0,187$ & 0,229 \\
\hline HbA1c & $-0,075$ & 0,632 \\
\hline Glucose & 0,157 & 0,315 \\
\hline HOMA-IR & $-0,104^{+}$ & 0,507 \\
\hline Insulin & $-0,112^{+}$ & 0,475 \\
\hline
\end{tabular}

Pearson's correlation analysis $\quad{ }^{+}$Spearman Rho correlation analysis $r$ : correlation coefficient

\section{Discussion}

Obesity is a general risk factor for insulin resistance, hypertension, glucose intolerance, hepatic steatosis, atherogenic dyslipidemia and type 2 diabetes mellitus (14). During the development of obesity, macrophages permeate into white adipose tissue and secrete various proinflammatory cytokines and chemokines along with adipocytes $(15,16)$. Galectin-3 plays an important role in the development of inflammation by interacting with various cytokines and chemokines (17). In an experimental study on Galectin-3 knock-out mice, it was reported that while in vivo galectin-3 administration resulted in glucose intolerance and insulin resistance; in vitro treatment with galectin-3 inhibitors might reduce insulin sensitivity directly on myocytes, hepatocytes and adipocytes (18). In addition, the protective role of galectin-3 has been mentioned in some studies $(8,9)$. Moreover, galectin-3 binds to advanced glycation end products that cause tissue damage related to the severity of diabetic complications and it has been reported that it protects the tissue damage due to stimulating their breakdown (19).
In our study, we investigated serum galectin-3 levels in adolescents for the first time in the literature. When we looked at the other studies related to this subject, we observed that the studies were generally on the experimental level, while a few studies were conducted in humans. Weigert et al. reported high levels of serum galectin- 3 in obese and type 2 diabetic patients (7). In the study of Rhodes et al., they showed that the expression of galectin-3 increased in visceral and subcutaneous adipose tissue in obese mice induced by high fat diet (20). Yilmaz et al. reported that high levels of galectin observed in prediabetes and diabetes resulted in development of diabetes and its complications (21). Li et al. reported that galectin-3 levels were increased in obesity and it was a proinflammatory molecule that would cause insulin resistance (18). In our study, we found that serum galectin-3 levels of obese adolescents were not statistically significantly different compared to normal weighted healthy adolescents. Since this is the first study in the literature evaluating the serum galectin-3 levels in the adolescent patients, we did not have the 
opportunity to compare our results directly with previous literature.

In our study, we also investigated the correlations between galectin-3 levels and parameters related to obesity and glucose metabolism. We found no statistically significant correlation between galectin-3 levels and BMI, glucose, insulin or HOMA-IR levels in the obese adolescent group. Weigert et al. reported a positive correlation between galectin-3 levels and $\mathrm{BMI}$ in type 2 diabetes mellitus and a negative correlation with $\mathrm{HbA1c}$ (7). Dencker et al. reported a positive correlation between galectin-3 levels and BMI in a study on nonobese children (22). Ohkura et al. reported that low galectin-3 levels were associated with insulin resistance (insulin levels and HOMA-IR) in type 2 diabetes but not with BMI (23). A negative correlation was reported between galectin-3 and HOMA-IR levels by $L i$ et al (18). It can be seen that the results of correlation analyzes in the previous studies are contradictory.

To discover the potential relationships between $\mathrm{BMI}$ and other markers against galectin-3 at a young age is important because of the increased risk of developing obesity in adulthood and the likelihood of serious short-term and long-term health problems in obese children and adolescents $(24,25)$. In this respect, our study data in adolescents makes it difficult to establish a relationship between the levels of obesity and galectin-3 observed in this group. This may be explained by the difference in the serum galectin-3 pattern in adolescents. Because obese adults are often decompensated by glucose metabolism, but obese children often have an early phase of alteration in glucose metabolism (26). In addition, we did not measure the levels of inflammatory cytokines in this study. Also, we did not analyze the galectin-3 levels at different stages of obesity which are the limitations of this study. Since galectin-3 plays a role in the development of inflammation by interacting with various cytokines $(5,6,17)$, we believe that the cytokine pattern in obesity may have a different effect than the expected on the galectin-3 synthesis, which may also influence our results.

In conclusion, we did not find any correlation between serum galectin-3 levels and obesity in adolescents. We believe that the effects of galectin-3 levels on obesity in adolescents should be analyzed in further investigations, which measures other biomarkers of the mechanism of action.

\section{Acknowledgments:}

This research did not receive any specific grants from any funding agency in the public, commercial, or not-for-profit sector.

\section{References:}

1. Dumic J, Dabelic S, Flo" gelM2006 Galectin3: an open-ended story. Biochim Biophys Acta 1760: 616-635.

2. Kiwaki K, Novak CM, Hsu DK, Liu FT, Levine JA 2007 Galectin-3 stimulates preadipocyte proliferation and is up-regulated in growing adipose tissue. Obesity (Silver Spring) 15: 32-39.

3. Rasouli N, Kern PA 2008 Adipocytokines and the metabolic complications of obesity. J Clin Endocrinol Metab 93: S64-S73.

4. Salvatore Sciacchitano, Luca Lavra, Alessandra Morgante, Alessandra Ulivieri, Fiorenza Magi, Gian Paolo De Francesco, Carlo Bellotti, Leila B. Salehi and Alberto Ricci. Galectin-3: One Molecule for an Alphabet of Diseases, from A to Z. Int. J. Mol. Sci. 2018, 19, 379; doi:10.3390/ijms1902 0379.

5. Pang J, Nguyen VT, Rhodes DH, Sullivan ME, Braunschweig C, Fantuzzi G. Relationship of galectin-3 with obesity, IL-6, and CRP in women. J Endocrinol Invest. 2016 Dec;39(12): 1435-1443.

6. Sabrina Krautbauer, Kristina Eisinger, Yvonne Hader, Christa Buechler. Free fatty acids and IL-6 induce adipocyte galectin-3 which is increased in white and brown adipose tissues of obese mice. Cytokine 69 (2014) 263-271. 
7. Weigert J, Neumeier $M$, Wanninger J, Bauer $\mathrm{S}$, Farkas S, Scherer $\mathrm{MN}$, et al. Serum galectin-3 is elevated in obesity and negatively correlates with glycosylated hemoglobin in type 2 diabetes. J Clin Endocrinol Metab 2010;95:1404-1411.

8. Pang J, Rhodes $\mathrm{DH}$, Pini $M$, Akasheh RT, Castellanos KJ, Cabay RJ, et al. Increased adiposity, dysregulated glucose metabolism and systemic inflammation in Galectin-3 KO mice. PloS One 2013; 8:e57915.

9. Pejnovic N, Pantic J, Jovanovic I, Radosavljevic G, Milovanovic M, Nikolic I, et al. Galectin-3 deficiency accelerates high-fat diet induced obesity and amplifies inflammation in adipose tissue and pancreatic islets. Diabetes 2013;62: 193244.

10. Martinez-Martinez E, Cachofeiro V, Rousseau E, Alvarez V, Calvier L, FernandezCelis $A$, et al. Interleukin-33/ST2 system attenuates aldosterone-induced adipogene sis and inflammation. Mol Cell Endocrinol 2015;411: 20-27.

11. Gómez García $A$, Núñez GG, Sandoval ME, Castellanos SG, Alvarez Aguilar C. Factors associated with early platelet activation in obese children. Clin Med Res. 2014;12: 21-6.

12. Mazıcıoglu MM, Hatipoğlu $N$, Öztürk $A$, Waist Circumference and Mid-Upper Arm Circumference in Evaluation of Obesity in Children Aged Between 6 and 17 Years. J Clin Res Ped Endo 2010;2(4): 144-150.

13. Matthews DR, Hosker JP, Rudenski AS, Naylor BA, Treacher DF, Turner RC. Homeostasis model assessment: insulin resistance and beta-cell function from fasting plasma glucose and insulin concentrations in man. Diabetologia 1985;28: 412-19.

14. Shoelson, S.E.; Lee, J.; Goldfine, A.B. Inflammation and insulin resistance. J. Clin. Investig. 2006, 116, 1793-1801.

15. Van Greevenbroek, M.M.; Schalkwijk, C.G.; Stehouwer, C.D. Obesity-associated lowgrade inflammation in type 2 diabetes mellitus: Causes and consequences. Neth. J. Med. 2013, 71, 174-187.

16. $\mathrm{Xu}$, L.; Kitade, H.; Ni, Y.; Ota, T. Roles of chemokines and chemokine receptors in obesity-associated insulin resistance and nonalcoholic fatty liver disease. Biomolecules 2015, 5, 1563-1579.

17. Calvier L, Miana M, Reboul $P$, Cachofeiro $V$, Martinez-Martinez E, de Boer RA, Poirier F, Lacolley $\mathrm{P}$, Zannad F, Rossignol P, LópezAndrés N (2013) Galectin-3 mediates aldosterone-induced vascular fibrosis. Arterioscler Thromb Vasc Biol 33(1): 67-75.

18. Li, P.; Liu, S.; Lu, M.; Bandyopadhyay, G.; Oh, D.; Imamura, T.; Johnson, A.M.F.; Sears, D.; Shen, Z.; Cui, B.; et al. Hematopoieticderived Galectin-3 Causes Cellular and Systemic Insulin Resistance. Cell 2016, 167, 973-984.

19. Zhu W, Sano H, Nagai R, Fukuhara K, Miyazaki A, Horiuchi S: The role of galectin-3 in endocytosis of advanced glycation end products and modified low density lipoproteins. Biochem Biophys Res Commun 2001, 280: 1183-1188.

20. Rhodes $\mathrm{DH}$, Pini $\mathrm{M}$, Castellanos $\mathrm{KJ}$, et al. Adipose tissue-specific modulation of galectin expression in lean and obese mice: evidence for regulatory function. Obesity. 2013;21(2): 310-319.

21. H. Yilmaz - M. Cakmak · O. Inan - T. Darcin · A. Akcay. Increased levels of galectin-3 were associated with prediabetes and diabetes: new risk factor?. J Endocrinol Invest (2015) 38: 527-533.

22. Magnus Dencker1 \& Daniel Arvidsson1,2 \& Magnus K. Karlsson3 \& Per Wollmer1 \& Lars B. Andersen4,5 \& Ola Thorsson. Galectin-3 levels relate in children to total body fat, abdominal fat, body fat distribution, and cardiac size. European Journal of Pediatrics (2018) 177: 461-467.

23. Ohkura T, Fujioka $Y$, Nakanishi $R$, Shiochi $H$, et al. 2014. Low serum galectin-3 concentrations are associated with insulin resistance in patients with type 2 diabetes mellitus. Diabetol Metab Syndr 6: 106. 
24. Alberdi G, McNamara $A E$, Lindsay $K L$, Scully HA, Horan MH, Gibney ER, McAuliffe FM (2016) The association between childcare and risk of childhood overweight and obesity in children aged 5 years and under: a systematic review. Eur J Pediatr 175(10): 1277-1294.

25. Gibson LY, Allen KL, Davis E, Blair E, Zubrick SR, Byrne SM (2017) The psychosocial burden of childhood overweight and obesity: evidence for persisting difficulties in boys and girls. Eur J Pediatr 176(7): 925-933. 27. Saner C, Simonetti GD, Wühl E, Mullis PE, Janner M (2016) Circadian and ultradian cardiovascular rhythmicity in obese children. Eur J Pediatr 175(8): 1031-1038.

26. Saner C, Simonetti GD, Wühl E, Mullis PE, Janner M (2016) Circadian and ultradian cardiovascular rhythmicity in obese children. Eur J Pediatr 175(8): 1031-1038. 